\title{
FOURIER SERIES ON THE RING OF INTEGERS IN A $p$-SERIES FIELD
}

\author{
BY MITCHELL H. TAIBLESON ${ }^{1}$
}

Communicated by A. P. Calderon, May 16, 1967

1. In this note we will describe a natural setting for harmonic analysis on the dyadic group, $2^{\omega}$, (also known as the Walsh-Paley group) and give a few illustrative results. Details and proofs will appear elsewhere.

The dyadic group is viewed classically as the set of all sequences of zeroes and ones with addition $(\bmod 2)$ defined pointwise, and is supplied with the usual product topology. From our point of view, $2^{\omega}$ will be the additive subgroup of the ring of formal power series in one variable over $G F(2)$.

The subject of this note is harmonic analysis on the ring of integers, $\mathfrak{O}$, in the field, $K$ (called a $p$-series field), of formal Laurent series (with finite principal part) in one variable over $G F(p)$, where $p$ is a prime. Such a field $K$ is a particular instance of a local field; that is, a locally compact, totally disconnected, nondiscrete, complete field. The $p$-adic fields are other examples of local fields. The results in this note have extensions to Fourier series on the ring of integers in any local field and also to multiple Fourier series. These extensions will not be given here.

The idea that $2^{\omega}$ might be an instance of a ring of integers in a local field developed in a conversation with E. M. Stein.

2. Let the prime $p$ be fixed. An element $x \in K$ is represented as $x=\sum_{-\infty}^{+\infty} a_{\nu} \mathfrak{p}^{\nu}, a_{\nu}=0$ for $\nu$ small enough, or equivalently as $x=\sum_{k}^{\infty} a_{\nu} p^{\nu}$, $a_{\nu}=0,1, \cdots$, or $p-1$ (viewed as elements of $G F(p)$ ). Addition and multiplication is given by the usual operations in the ring of formal power series over $G F(p)$. A topology is given on $K$ by constructing basic neighborhoods $N_{x, k}=\left\{y=\sum b_{\nu} p^{\nu}: b_{\nu}=a_{\nu}, \nu<k\right\}$, for each $k \in \boldsymbol{Z}$ and $x=\sum a_{\nu} p^{\nu}$. With this topology $K$ is a locally compact, totally disconnected, nondiscrete, complete field.

We embed $G F(p)$ in $K$ in the obvious way. The series representations are then convergent series in $K$ and are unique.

The ring of integers $\mathfrak{D}=\left\{x: x=\sum_{\nu=0}^{\infty} a_{\nu} \mathfrak{p}^{\nu}\right\}$ is the unique maximal compact subring of $K$.

${ }^{1}$ Research partially supported by the Air Force Office of Scientific Research, Office of Aerospace Research, United States Air Force under AFOSR Contract No. AF 49(638)-1769 and Grant AF-AFOSR-1071-66. 
Let $d x$ be Haar measure on $K$ normalized so $|\supseteq|=1$. If $\alpha \in K^{*}$ define $|\alpha|$ by $d(\alpha x)=|\alpha| d x$. Let $|0|=0$. Then $\alpha \rightarrow|\alpha|$ is a nonarchimedian valuation on $K$.

The principal ideal in $\mathfrak{D}$ generated by $\mathfrak{p}, \mathfrak{P}$, is the unique maximal ideal in $\mathfrak{O}$. $\mathfrak{P}=\mathfrak{p} \mathfrak{D}=(\mathfrak{p})$. $|\mathfrak{P}|=|\mathfrak{p}|=p^{-1}$. Let $\mathfrak{D}^{*}=\mathfrak{D}-\mathfrak{B}=\{x:|x|$ $=1\}$. Any $x \in K^{*}$ can be written uniquely as $x=p^{k} x^{\prime}, x^{\prime} \in D^{*}$. It follows that $|x|=0$ iff $x=0$, and if $x \neq 0,|x|=p^{-k}$ for some integer $k$.

REMARK. If $x \in \mathfrak{D}, x \neq 0$, then $|x|=p^{-k}$ where $k$ is the number of zeroes that precedes the first nonzero coefficient in the series $x$ $=\sum_{0}^{\infty} a_{\nu} p^{\nu}$. With this formulation the function $x \rightarrow|x|$ was used by Harper [3] in his investigation of potentials on $2^{\omega}$.

The topology induced by the norm on $K$ agrees with the original topology.

Let $\mathfrak{P}^{k}=\left\{x:|x| \leqq p^{-k}\right\}$. Then $\mathfrak{P}^{1}=\mathfrak{P}, \mathfrak{P}^{0}=\mathfrak{O},\left|\mathfrak{P}^{k}\right|=p^{-k}\left(1-p^{-1}\right)$.

If $\chi$ is a continuous unitary character on $K^{+}$then $\chi(x)$ is a $p$ th root of unity for all $x \in K$ and $\chi$ is trivial on some $\mathfrak{S}^{k} . \chi$ is completely determined by specifying the values of $\chi\left(p^{\nu}\right)$ for all integers $\nu$. Any such specification with $p$ th roots of unity is possible provided $\chi\left(p^{\nu}\right)=1$ for $\nu$ large enough.

An alternate description is given by singling out any nontrivial character $\chi$ and then noticing that all other characters are of the form $\chi_{u}(x)=\chi(u x)$ for some $u \in K$. In fact, $u \rightarrow \chi_{u}$ is a topological isomorphism of $K^{+}$onto its dual group, and so we identify $K^{+}$with its dual group. In the sequel $\chi$ will represent some fixed character that is trivial on $\mathfrak{D}$, but is nontrivial on $\mathfrak{P}^{-1}$.

The Fourier transform is initially defined for $f \in L^{1}$ by $\hat{f}(u)$ $=\int_{K} f(x) \chi(-u x) d x$. The following facts play a crucial role.

LEMma A. If $f \in L^{1}$ and $f$ is supported on $\mathfrak{P}^{m}$ then $\hat{f}$ is constant on the cosets of $\mathfrak{B}^{-m}$ in $K^{+}$. If $f \in L^{1}$ and is constant on the cosets of $\mathfrak{P}^{n}$, then $\hat{f}$ is supported on $\mathfrak{B}^{-n}$. Hence if $\Phi_{k}$ is the characteristic function of $\mathfrak{P}^{-k}$, then $p^{-k} \hat{\Phi}_{k}=\Phi_{-k}$.

The linear spaces of test functions $\subseteq$ and tempered distributions $\Im^{\prime}$ and the Fourier transform on $\Im^{\prime}$ are defined as in [5]. For details see $[5, \S 2]$ and $[4]$.

3. $\mathfrak{D}$ is a locally compact abelian group under addition. $\mathfrak{S}$ can be identified with $K / \mathfrak{D}$ and every character on $\mathfrak{D}$ is of the form $\chi_{u}$ restricted to $\mathfrak{O}$ where $u$ is a coset representative of $\mathfrak{O}$ in $K$. By choosing the representatives to be 0 or of the form $\sum_{s=1}^{k} r_{s} p^{-s}$ and using the usual lexicographic ordering the characters on $\mathfrak{D}$ are matched to the nonnegative integers. The correspondence $\chi_{n} \leftrightarrow \chi_{u(n)}$ satisfies: $n$ $\leqq|u(n)| \leqq p n$. 
If $n$ and $m$ are nonnegative integers whose $p$-adic expansions have disjoint nonzero entries then $\chi_{n+m}=\chi_{n} \chi_{m}$. For all $x \in K$ and nonnegative integers $n$ we have $\chi_{n}\left(p^{-s} x\right)=\chi_{p^{s_{n}}}(x)$ if $s$ is a nonnegative integer. The sequence $\left\{\chi_{p^{k}}\right\}_{k=1}^{\infty}$ is the exact analogue of the Rademacher functions.

REMARK. In Fine's basic work on Walsh functions [1] he made essential use of a measure preserving map between $2^{\omega}$ and $[0,1]$. He needed a relation analogous to $\chi_{n}\left(p^{-8} x\right)=\chi_{n \cdot p^{8}}(x)$ and moved over to the reals to perform the required multiplication. Using the ring structure of $\mathfrak{O}$ we may work in $\mathfrak{O}$ and $K$, exploiting the unexpected fact that in a group of characteristic $p$, there is an element, $p$, that "multiplies by $p^{-1}$."

Let $\subseteq(\mathfrak{D})$ be the linear space of restrictions of members of $\subseteq$ to $\mathfrak{N}$, with the obvious quotient topology. Then $\mathfrak{S}^{\prime}(\mathfrak{O})$, the topological dual of $\mathfrak{S}(\mathfrak{D})$ (called the space of distributions on $\mathfrak{D}$ ) consists of the restrictions of the members of $\mathfrak{S}^{\prime}$ to $\mathfrak{D}$. If $f \in \mathbb{S}^{\prime}(\mathfrak{D})$ and $\phi \in \mathfrak{S}(\mathfrak{D})$ then there are $f^{*} \in \mathfrak{S}^{\prime}, \phi^{*} \in \mathfrak{S}$ such that $f=f^{*} \Phi_{0}, \phi=\phi^{*} \Phi_{0}$ on $\mathfrak{D}$, and the action of $f$ on $\phi$ is given by $\langle f, \phi\rangle=\left\langle f^{*}, \phi\right\rangle=\left\langle f, \phi^{*}\right\rangle$, where either of the last two terms defines the first.

If $f \in \mathscr{S}^{\prime}(\mathfrak{D})$ then the $n$th Fourier coefficient of $f$ is defined as $c_{n}=\left\langle f, \chi_{n}(-x) \Phi_{0}\right\rangle$. When $f$ is integrable on $\mathfrak{O}$ this reduces to $c_{n}$ $=\int_{\mathscr{O}} f(x) \chi_{n}(-x) d x$. For $f \in \mathbb{S}^{\prime}(\mathfrak{D})$ we write $f \sim \sum_{n=0}^{\infty} c_{n} \chi_{n}$ and call the right-hand side the Fourier series of $f$.

If $f \in L^{1}(\supseteq)$ and is defined on all of $K$ by setting it equal to zero outside of $\mathfrak{D}$, then $f \in L^{1}(K)$ and by Lemma $A, \hat{f}$ is constant on the cosets of $\mathfrak{D}$ in $K$. It can be seen that $c_{n}=\hat{f}(u(n))$ for all $n$.

The Dirichlet kernels are defined by $D_{n}(x)=\sum_{s=0}^{n-1} \chi_{n}$, so that

$$
\begin{aligned}
s_{n}(x ; f) & =\sum_{s=0}^{n-1} c_{n} \chi_{n}(x)=\int_{y \in \mathcal{D}} f(y) D_{n}(x-y) d x \\
& =\int_{y \in \mathcal{D}} f(x-y) D_{n}(y) d y, x \in \mathfrak{D}
\end{aligned}
$$

are the partial sums for $f$ when $f$ is integrable on $\mathfrak{D}$.

A basic fact is:

TheOREM 1. $D_{p^{n}} \Phi_{0}=p^{n} \Phi_{-n}, n=0,1,2, \cdots$,

$$
s_{p^{n}}(x ; f)=p^{n} \int_{|x-y| \leqslant p^{-n}} f(y) d y, \quad x \in \mathcal{D}, f \in L^{1}(\mathfrak{D}) .
$$

As an easy consequence we have: 
Corollary 2. (a) If $f \in L^{1}(\mathfrak{D}), s_{p^{n}}(x ; f) \rightarrow f(x)$ a.e. as $n \rightarrow \infty$. In particular $s_{p^{n}}(x ; f) \rightarrow f(x)$ at each point of continuity of $f$.

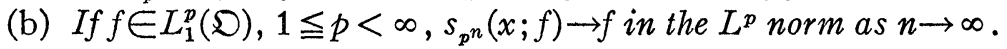

(c) If $f$ is continuous on $\mathfrak{D}, s_{p^{n}}(x ; f) \rightarrow f$ uniformly as $n \rightarrow \infty$.

4. To simplify some of our considerations we will fix on a particular $\chi$. Specifically we let $\chi\left(\mathfrak{p}^{\nu}\right)=1$ if $\nu \geqq 0$, and $\chi\left(\mathfrak{p}^{\nu}\right)=\delta$ if $\nu<0$, where $\delta$ is a fixed primitive $p$ th root of unity. Any other character that is trivial on $\mathfrak{D}$, but nontrivial on $\mathfrak{P}^{-1}$ will be of the form $\chi_{u}$ for some $u \in \mathfrak{S}^{*}$. That is, it will be a rotation of $\chi$.

From another point of view the orthonormal sequence $\left\{\chi_{n}(u x) \Phi_{0}\right\}_{n=0}^{\infty}$ is obtained from $\left\{\chi_{n} \Phi_{0}\right\}_{n=0}^{\infty}$ by a permutation that leaves the blocks $\left\{\chi_{n} \Phi_{0}\right\}_{n=p^{t}}^{p^{t+1}}$ fixed as a set for $t=0,1,2, \cdots$, and leaves $\chi_{0}$ fixed. There are exactly as many permissible permutations of the elements in a block as there are elements in that block. For example, the possible arrangements of $\left\{\chi_{1}, \chi_{2}, \cdots, \chi_{p-1}\right\}$ are given by $c, 2 c, \cdots$, $(p-1) c$, where $c=1,2, \cdots, p-1$ and the multiplication is taken modulo $p$.

5. We now consider the Fejér kernels, $K_{n}$;

$$
K_{n}=1 / n \sum_{r=1}^{n} D_{r}=\sum_{r=0}^{n-1}(1-r / n) \chi_{r} .
$$

For $|x|=p^{k}$ let $x^{\prime}=p^{-k} x$, and if $x=0$ let $x^{\prime}=0$. We can show that:

LEMMA 3.

$$
\begin{aligned}
\text { (a) } \quad \begin{aligned}
K_{p^{n}}(x) & =\left(p^{n}+1\right) / 2, \quad \text { if }|x| \leqq p^{-n}, \\
= & p^{n-s}\left\{\left(1-p^{-1}\right) K_{p-1}(x)\right\}\left\{p^{1-s} D_{p^{s-1}}\left(p^{-1} x^{\prime}\right)\right\}
\end{aligned} \\
\quad \text { if }|x|=p^{-n+s}, s=1,2, \cdots, n . \\
\text { (b) } \quad\left|\left(1-p^{-1}\right) K_{p-1}(x)\right| \leqq(p-1) / 2, \quad x \in \mathfrak{D}^{*}, \\
\operatorname{Re}\left\{\left(1-p^{-1}\right) K_{p-1}(x)\right\}=1 / 2, \quad x \in \mathfrak{D}^{*} . \\
\text { (c) Let } x_{1}=1+(p-1) \mathfrak{p}, \quad x_{x}=s x_{1}, \quad s=2,3, \cdots, p-1 .
\end{aligned}
$$

Then the restriction of $p^{1-8} D_{p^{s-1}}\left(p^{-1} x\right)$ to $\mathfrak{D}^{*}$ is the characteristic function of $\bigcup_{t=1}^{p-1} x_{t}+\mathfrak{P}^{t}$.

These relations are consequences of the fact that for all $x$ and $n$, $\left\{\chi_{p^{n}}(x), x_{2-p^{n}}(x), \cdots, \chi_{(p-1) p^{n}}(x)\right\}$ is a sequence of powers of a $p$ th root of unity and that for suitably related $n$ and $m, \chi_{n+m}=\chi_{n} \chi_{m}$.

We then get the consequences of Corollary 2 for $\sigma_{p^{n}}(x ; f)$ using a local field variant of a lemma of Fine for part a. 
REMARK. If $p=2$ then $K_{p-1}(x) \equiv 1$ for $x \in \mathcal{D}$ and so $K_{2^{n}}(x) \geqq 0$ for all $n$ and $x \in \mathfrak{D}$.

A suitable generalization of Fine's argument, using the facts that for $r=1,2, \cdots, p-1, D_{r p^{n}}(x)=D_{p^{n}}(x) D_{r}\left(p^{-n} x\right)$ and $r p^{n} K_{r p^{n}}(x)$ $=p^{n} D_{p^{n}}(x)(r-1) K_{r-1}\left(p^{-n} x\right)+p^{n} K_{p^{n}}(x) D_{r}\left(p^{-n} x\right)$ yields:

TheOREM 4. The conclusion of Corollary 2 for $\sigma_{n}(x ; f)$.

6. From the behavior of the factors $p^{1-s} D_{p^{s-1}}\left(p^{-1} x^{\prime}\right)$ we derive the following:

Definition. We say that the limit of $f$ at $x$ in the direction $s$ $=1,2, \cdots$, or $p-1$ exists if $f(x+s \cdot 0)=\lim _{k \rightarrow \infty} f(x+h)$ exists

$$
h \in \mathfrak{p}^{k}(s+\mathfrak{P}) \text {. }
$$

TheOREM 5. If $f \in L^{1}(\mathfrak{D}), f(x+s \cdot 0)$ exists for $s=1,2, \cdots, p-1$, then $\sigma_{n}(x ; f) \rightarrow(p-1)^{-1} \sum_{s=1}^{p-1} f(x+s \cdot 0)$ as $n \rightarrow \infty$.

7. Suppose $f$ is a distribution on $K$. Then

TheOREM 6. $f \Phi_{0} \sim \sum\left(\hat{f}^{*} \Phi_{0}\right)(u(n)) \chi_{n}$.

This is essentially the Poisson Summation formula.

If $f \Phi_{0} \in L^{1}$ and $\hat{f}$ is locally integrable we obtain

Corollary 7. $\int_{|x| \leq 1} f(x) \chi(-u x) d x=\int_{|x| \leq 1} \hat{f}(u-x) d x, u \in K$.

We say that $f$ is locally constant at $x$ if $f$ is constant on $x+\mathfrak{B}^{k}$ for some $k$. It can be seen that if $f$ is integrable on $\mathfrak{D}$ and is locally constant at $x$ then $f(x)=\sum c_{n} \chi_{n}(x)$.

Suppose now that $f$ is a radial function on $K(f(x)$ depends only on $|x|), f \Phi_{0}$ is integrable, $\hat{f}$ is locally integrable. Then $\hat{f}$ is radial and $f$ is locally constant at all $x \neq 0$. Since $\hat{f}$ is radial we see that $\left(\hat{f}^{*} \Phi_{0}\right)(u)$ $=\hat{f}(u)$ if $|u|>1$. Let $c_{0}=\left(\hat{f}^{*} \Phi_{0}\right)(0)=\int_{|x| \leq 1} \hat{f}(x) d x=\int_{|x| \leq 1} f(x) d x$. We conclude

$$
f \Phi_{0} \sim c_{0}+\sum_{n \neq 0} \hat{f}(u(n)) \chi_{n}
$$

and

$$
f(x)=c_{0}+\sum_{n \neq 0} \hat{f}(u(n)) \chi_{n}(x), \quad 0<|x| \leqq 1 .
$$

A case of interest is the collection of potential kernels, $f_{\alpha}(x)=|x|^{-\alpha}$, $0<\alpha<1$. In [5] it was shown that $\hat{f}_{\alpha}(u)=1 / \Gamma_{1}(\alpha)|u|^{\alpha-1}$ where $\Gamma_{1}(\alpha)=\left(1-p^{\alpha-1}\right) /\left(1-p^{-\alpha}\right)$. For the $\alpha=0$ case, we want log $(1 /|x|)$. In [7] we showed that $G_{1}(x)=\left\{(\log (p /|x|))\left(1-p^{-1}\right) / \log p\right\} \Phi_{0}$, where $\hat{G}_{1}(u)=(\max (1,|x|))^{-1}$. 
These functions satisfy the requirements of the preceding paragraph and so

$$
\begin{array}{r}
|x|^{-\alpha} \Phi_{0} \sim\left(1 / \Gamma_{1}(\alpha)\right)\left\{\left(1-p^{-1}\right) /\left(1-p^{-\alpha}\right)+\sum_{n \neq 0}|u(n)|^{\alpha-1} \chi_{n}\right\}, \\
|x|^{-\alpha}=1 / \Gamma_{1}(\alpha)\left\{\left(1-p^{-1}\right) /\left(1-p^{-\alpha}\right)+\sum_{n \neq 0}|u(n)|^{\alpha-1} \chi_{n}(x)\right\}, \\
0<|x| \leqq 1
\end{array}
$$

and

$$
\begin{aligned}
& \log (p /|x|) \Phi_{0}=\log \left(p /\left(1-p^{-1}\right)\right)\left\{1+\sum_{n \neq 0}|u(n)|^{-1} \chi_{n}\right\}, \\
& \log (1 /|x|) / \log p=\left(1-p^{-1}\right)^{-1}\left\{1 / p+\sum_{n \neq 0}|u(n)|^{-1} \chi_{n}(x)\right\}, \\
& 0<|x| \leqq 1 .
\end{aligned}
$$

These relations were obtained for $2^{\omega}$ by Harper [3].

8. The notion of a function of bounded variation has been studied on groups related to $\mathfrak{O}$ at several junctures. It has been looked at in the particular instance of $2^{\omega}[1]$ and in the more general setting of the duals of countable, periodic, abelian groups [8]. The earlier approaches have used a natural map from the group to $[0,1]$ and then used the order imposed on the group by this map to define the variation of a function. One purpose of these investigations has been to obtain the analogue of the classical result that the Fourier coefficients of a function of bounded variation are $O(1 / n)$. We will give an orderfree definition of a class of functions with this property.

Definition. A partition of $\mathfrak{D}$ is a finite collection $\Pi=\left\{S_{k}\right\}_{k=1}^{n}$ such that $S_{k}=x_{k}+\mathfrak{P}^{v_{k}}$ for some $x_{k} \in \mathfrak{D}, \nu_{k} \geqq 0, S_{k} \cap S_{l}=\varnothing$ if $k \neq l$ and $\mathfrak{D}=\bigcup_{k} S_{k}$.

Let $v_{k}(\Pi)=\sup _{x \in S_{k}} f(x)-\inf _{x \in S_{k}} f(x)$, where we assume that $f$ is real valued. Let $V_{\pi}=\Sigma_{k} v_{k}(\Pi), V=\sup _{\pi} V_{\pi}$.

Definition. We say that $f$ is of bounded variation on $\mathfrak{O}$ if $V(\operatorname{Re} f)$ $+V(\operatorname{Im} f)<\infty$ and define $V(f)=V(\operatorname{Re} f)+V(\operatorname{Im} f)$.

REMARK. This notion has its roots in Study's notion of a "function of bounded fluctuation," which he showed was equivalent to bounded variation [6].

It can be easily seen that if $f$ is of bounded variation on $\mathfrak{O}$, then it is continuous except on a countable set. 
THEOREM 8. If $f$ is of bounded variation on $\bigcirc$ and $f \sim \Sigma c_{n} \chi_{n}$ then $c_{n}=O(1 / n)$ as $n \rightarrow \infty$.

This notion of bounded variation is strictly weaker than the one using the order of $[0,1]$.

Using Theorem 4, Theorem 5 , and Theorem 8 we obtain:

THEOREM 9. (a) If $f$ is of bounded variation on $\mathfrak{D}$ then $s_{n}(x: f) \rightarrow f(x)$ for all $x$ not in the exceptional countable set where $f$ is not continuous.

(b) At each $x \in \mathfrak{O}$ where $f(x+s \cdot 0)$ exists for $s=1,2, \cdots, p-1$, then $s_{n}(x: f) \rightarrow(p-1)^{-1} \sum_{s=1}^{p-1} f(x+s \cdot 0)$.

\section{REFERENCES}

1. N. J. Fine, On the Walsh functions, Trans. Amer. Math. Soc. 65 (1949), 372-414.

2. Cesaro summability of Walsh-Fourier series, Proc. Nat. Acad. Sci. U.S.A. 41 (1955), 588-591.

3. L. H. Harper, Capacities of sets and harmonic analysis on the group $2^{\omega}$, Trans. Amer. Math. Soc. 126 (1967), 303-315.

4. K. L. Phillips, Hilbert transforms for the p-adic and p-series fields, Pacific J. Math. (to appear).

5. P. J. Sally, Jr. and M. H. Taibleson, Special functions on locally compact fields, Acta Math. 116 (1966), 279-309.

6. E. Study, Ueber eine besondere Classe von Functionen einer reelen Verändlichen, Math. Ann. 47 (1896), 298-316.

7. M. H. Taibleson, Harmonic analysis on n-dimensional vector spaces over local fields. I, Basic results on fractional integration, (to appear).

8. N. Ja. Vilenkin, On a class of complete orthonormal systems, Amer. Math. Soc. Transl. (2) 28 (1963), 1-35.

WASHINGTON UNIVERSITY AND

The Institute for Advanced Study 